\title{
Characterization of the meningococcal DNA glycosylase Fpg involved in base excision repair
} Katrina L Tibballs ${ }^{1}$, Ole Herman Ambur ${ }^{2}$, Kristian Alfsnes ${ }^{2}$, Håvard Homberset ${ }^{1}$, Stephan A Frye ${ }^{1,2}$, Tonje Davidsen ${ }^{1}$ and Tone Tønjum*1,2

Address: ${ }^{1}$ Centre for Molecular Biology and Neuroscience and Institute of Microbiology, University of Oslo, Rikshospitalet, NO-0027 Oslo, Norway and 2Institute of Microbiology, Rikshospitalet, NO-0027 Oslo, Norway

Email: Katrina L Tibballs - k.l.tiballs@medisin.uio.no; Ole Herman Ambur - ole.herman.ambur@rr-research.no; Kristian Alfsnes - kristian.alfsnes@rr-research.no; Håvard Homberset - havard.homberset@ rr-research.no; Stephan A Frye - stephan.frye@rrresearch.no; Tonje Davidsen - tonje.davidsen@rr-research.no; Tone Tønjum* - tone.tonjum@rr-research.no

* Corresponding author

Published: 9 January 2009

BMC Microbiology 2009, 9:7 doi:10.1 |86/147|-2/80-9-7
Received: 9 September 2008

Accepted: 9 January 2009

This article is available from: http://www.biomedcentral.com/I47I-2/80/9/7

(C) 2009 Tibballs et al; licensee BioMed Central Ltd.

This is an Open Access article distributed under the terms of the Creative Commons Attribution License (http://creativecommons.org/licenses/by/2.0), which permits unrestricted use, distribution, and reproduction in any medium, provided the original work is properly cited.

\begin{abstract}
Background: Neisseria meningitidis, the causative agent of meningococcal disease, is exposed to high levels of reactive oxygen species inside its exclusive human host. The DNA glycosylase Fpg of the base excision repair pathway (BER) is a central player in the correction of oxidative DNA damage. This study aimed at characterizing the meningococcal Fpg and its role in DNA repair.

Results: The deduced N. meningitidis Fpg amino acid sequence was highly homologous to other Fpg orthologues, with particularly high conservation of functional domains. As for most N. meningitidis DNA repair genes, the $\mathrm{fpg}$ gene contained a DNA uptake sequence mediating efficient transformation of DNA. The recombinant N. meningitidis Fpg protein was over-expressed, purified to homogeneity and assessed for enzymatic activity. N. meningitidis Fpg was found to remove 2,6diamino-4-hydroxy-5-formamidopyrimidine (faPy) lesions and 7,8-dihydro-8-oxo-2'deoxyguanosine (80xoG) opposite of $\mathrm{C}, \mathrm{T}$ and $\mathrm{G}$ and to a lesser extent opposite of $\mathrm{A}$. Moreover, the $N$. meningitidis fpg single mutant was only slightly affected in terms of an increase in the frequency of phase variation as compared to a mismatch repair mutant.
\end{abstract}

Conclusion: Collectively, these findings show that meningococcal Fpg functions are similar to those of prototype Fpg orthologues in other bacterial species.

\section{Background}

Neisseria meningitidis, or the meningococcus (Mc), exclusively colonizes the oro- and nasopharynx of humans. It resides as a commensal in approximately $10 \%$ of healthy individuals [1], but may become virulent, disseminating into the bloodstream and crossing the blood-brain barrier
[2]. Mc septicaemia and meningitis are the cause of significant morbidity and mortality worldwide [2].

On the mucosal surface of the upper respiratory tract, Mc is exposed to reactive oxygen species (ROS) produced by the immune system through the oxidative burst and by 
endogenous aerobic metabolism, causing damage to many cellular components, including DNA [3]. Oxidative DNA lesions comprise single- and double strand breaks, abasic (apurinic/apyrimidinic, or AP) sites, and base damages, among which one of the most common is the oxidation product of guanine, 7,8-dihydro-8-oxo-2'deoxyguanosine (8oxoG). The mutagenic 8oxoG can mispair with adenine during replication and cause $\mathrm{G}: \mathrm{C} \rightarrow \mathrm{T}: \mathrm{A}$ transversions [4]. 2,6-diamino-4-hydroxy-5-formamidopyrimidine (faPy) is another oxidative modified form of guanine that inhibits DNA synthesis [5].

The base excision DNA repair pathway (BER) is the main defense against the mutagenic and cytotoxic effects of endogenously damaged bases. This enzymatic pathway has been identified in all organisms studied to date [6]. A DNA glycosylase initiates this pathway by cleaving the glycosylic bond between its specific base substrate and the sugar-phosphate backbone, leaving an abasic (AP) site [6]. Many DNA glycosylases also have an inherent AP lyase activity that cleaves the sugar-phosphate backbone at the AP site, which is subsequently repaired by further BER enzymes. In E. coli, formamidopyrimidine-DNA glycosylase (Fpg) shows substrate specificity for 8oxoG and faPy lesions, and exhibits AP lyase activity, in successive $\beta$ and $\delta$-elimination steps, leaving a single strand break [7].

In E. coli, the mutagenic effects of oxidated guanines are prevented by a triplet of enzymes termed the GO system [8]. In GO, Fpg acts together with the DNA glycosylase MutY which removes adenine when mispaired with 8oxoG, and MutT, a nucleotide hydrolase that converts 8oxoGTP to 8oxoGMP, preventing incorporation of oxidized GTPs into the genomic DNA. Mc single fpg mutants only elicit a weak mutator phenotype [9], however, mutYfpg double mutants exhibit a much higher increase in spontaneous mutation frequency than would be expected if $f p g$ and $m u t Y$ were involved in unrelated repair mechanisms [9]. This synergistic effect of the two Mc DNA glycosylases confirms their essential role in the repair of oxidative DNA damage and a relationship similar to that in the E. coli GO system. In vivo Mc Fpg activity has previously been detected in whole cell extracts of clinical isolates by cleavage of 8 oxoG opposite $\mathrm{C}[10]$, however, the Mc Fpg substrate specificity has not previously been investigated.

In this study, the Mc fpg gene was cloned and its gene product over-expressed and purified to homogeneity. Recombinant Mc Fpg was assessed with regard to its enzymatic activity towards recognized Fpg DNA substrates. The Mc MC58 Fpg DNA sequence [11], flanking regions and predicted amino acid sequence was analyzed. Furthermore, sequences of $f p g$ homologues and flanking regions in other neisserial species were aligned and examined. Finally, an Mc fpg mutant was assessed with regard to phase variation rate and compared to that of the wildtype strain and mismatch repair defective mutants. In essence, the Mc Fpg predicted structure and the activity pattern detected were similar to those of prototype Fpg orthologues in other species.

\section{Methods \\ Bacterial strains, plasmids, and DNA manipulations}

Bacterial strains and plasmids used in this study are listed in Table 1. DNA isolation, PCR amplification and cloning were performed according to standard techniques [12]. The fpg gene from Mc strain M1080 was PCR amplified using primers KT1b and KT2b (Table 2). The fpg-containing DNA fragment was cloned into the expression vector pET22b, creating plasmid pET22b-fpgM1080. E. coli ER2566 was used for pET22b-fpgM1080 plasmid propaga-

Table I: Bacterial strains and plasmids used in this study.

\begin{tabular}{|c|c|c|}
\hline Plasmid, strains & Relevant characteristic & Source/Reference \\
\hline \multicolumn{3}{|l|}{ Plasmids } \\
\hline pET22b & Expression vector, T7 promoter-driven system, His-tag, amp $p^{R}$ & Novagen \\
\hline pET22b-fpgMI080 & pET22b harbouring fpg from Mc MI080 & This study \\
\hline PARR2107 & Contains an Universal Rate Of Switching cassette & {$[22]$} \\
\hline PUD & PARR2 I07 harbouring a 12-mer DUS & This study \\
\hline \multicolumn{3}{|l|}{ Strains } \\
\hline \multicolumn{3}{|l|}{ Escherichia coli } \\
\hline \multirow[t]{2}{*}{ ER2566 } & Expression host with chromosomal copy of the T7 & New England \\
\hline & RNA polymerase gene & Biolabs \\
\hline ER2566-pET22b-fpgMI080 & ER2566 expressing Mc MI080 fpg from pET22b & This study \\
\hline \multicolumn{3}{|l|}{ Neisseria meningitidis } \\
\hline MI080 & Serogroup B, isolated in the USA in 1970 & {$[45]$} \\
\hline Z1099 & Serogroup A, isolated in the Philippines in 1968 & Dominique A. Caugant \\
\hline NmZI099_UROS & Z1099 harbouring a Universal Rate Of Switching cassette & This study \\
\hline NmZ1099_UROS $\triangle \mathrm{fpg}$ & Z1099 fpg strain harbouring a Universal Rate Of Switching cassette & This study \\
\hline NmZI099_UROSAmutS & Z1099 mutS strain harbouring a Universal Rate Of Switching cassette & This study \\
\hline
\end{tabular}


Table 2: The DNA sequences of primers used in this study.

\begin{tabular}{|c|c|c|c|}
\hline Oligonucleotide & Sequence $\left(5^{\prime}-3^{\prime}\right) *$ & Application & Source \\
\hline KTIb & gggaattccatatgcctgaattgccggaagtggaaacg & Cloning & This study \\
\hline $\mathrm{KT} 2 \mathrm{~b}$ & cgcgctcgagtttctgacagttcgggcaata & Cloning & This study \\
\hline TD 146 & gaagtggaaacgacgttgcgcg & Sequencing & This study \\
\hline TD 147 & cgtgccgcgetgccccaaagtttc & Sequencing & This study \\
\hline TD 160 & ctcataccaaagtatcgc & Sequencing & This study \\
\hline TDI6I & ttcgcccaccgtcctgc & Sequencing & This study \\
\hline TD46 & gctgttggaaaaactggg & Sequencing & This study \\
\hline TD47 & gcatacagataatccgtgc & Sequencing & This study \\
\hline spcFOR & cccagtggacataagcctgt & G-tract control, PCR/sequencing & {$[22]$} \\
\hline spcREV & agccgaagtttccaaaaggt & G-tract control, PCR/sequencing & {$[22]$} \\
\hline N248 & ggcggcatgaccc8oxoggaggcccatc & DNA substrate Containing 8oxoG lesion & Eurogen \\
\hline T248 & gatgggcctccgggtcatgccgcc & DNA substrate, complementary to N248 & Eurogen \\
\hline 1393 & gatgggcctcagggtcatgccgcc & DNA substrate, complementary to N248 & Eurogen \\
\hline 1394 & gatgggcctcggggtcatgccgcc & DNA substrate, complementary to N248 & Eurogen \\
\hline 1395 & gatgggcctctgggtcatgccgcc & DNA substrate, complementary to N248 & Eurogen \\
\hline $\mathrm{H} 7$ & aacaacaacaaatgccgtctgaaccaacatgccgtctgaaaacaacaacaac & Undamaged DNA substrate & This study \\
\hline $\mathrm{H} 8$ & gttgttgttgttttcagacggcatgttggttcagacggcatttgttgttgtt & Undamaged DNA substrate, complementary to H7 & This study \\
\hline
\end{tabular}

* letters in bold represents the DNA lesion or its complementary base in the DNA substrate

tion. DNA sequencing of pET22b-fpgM1080 was performed on a Beckman Coulter CEQ8000 Genetic Analyser System (Beckman Instruments, Ca) using an ABI BigDye Terminator v. 3.1 DNA sequencing kit (Applied Biosystems, NC), with primers listed in Table 2.

\section{Bioinformatics analyses and search for signature motifs}

An in silico search for functional domains and DNA binding motifs was carried out on the deduced amino acid sequence of Mc MC58 Fpg (NMB1295), using the the Expasy site http://us.expasy.org/cgi-bin/protscale.pl and the PROSITE [13] and Pfam databases [14]. The electrostatic charge was calculated using the EMBOSS package http://emboss.sourceforge.net/. The DNA sequences of the $f p g$ genes and flanking regions as well as the deduced amino acid sequences were aligned and compared in a panel of neisserial species for which the genome sequences were available. The following genome sequences (with accession numbers) were downloaded from Genbank http://www.ncbi.nlm.nih.gov/: Neisseria gonorrhoeae FA1090 (NC 002946), Mc MC58 serogroup B (NC 03112) [11], Mc Z2491 serogroup A ( [15], Mc FAM18 serogroup C (NC_03221) [16] and MC 053442 serogroup C (NC_010120) [17]. The temporary sequence data for Neisseria lactamica ST-640 was obtained from the Pathogen Sequencing Unit at the Sanger Institute ftp://ftp.sanger.ac.uk/pub/pathogens/nl/. Access to the genome sequence of Mc 8013 serogroup $\mathrm{C}$ was provided by Eduardo Rocha, ABI/Institut Pasteur, Paris, France, with kind permission from Vladimir Pelicic, Necker Hospital, Paris/Imperial College London. Prediction of the Fpg secondary structure was performed based on a blast search http://www.ncbi.nlm.nih.gov/blast/Blast.cgi in the JPred program [18]. Protein data bank (PDB) structural modeling was performed using SMART http:// smart.embl-heidelberg.de/, $\quad$ Pfam $\quad \underline{\text { http:// }}$ www.sanger.ac.uk/Software/Pfam/, Phyre http:// www.sbg.bio.ic.ac.uk/phyre/ and PyMol http:// www.pymol.org.

The Mc fpg flanking regions were searched for the presence of transcriptional terminators with the GeSTer genome scanner for terminators (the last version of the program is available at http://pallab.physics.iisc.ernet.in/ sum05/ anil/projects.html and the TransTermHP program http:// transterm.cbcb.umd.edu/. Operon predictions were taken from VIMSS [19]. Putative promoters were identified with the transcription promoter predictor available at the Berkeley Drosophila Genome Project http://www.fruit fly.org/seq tools/promoter.html and the BPROM predictor of bacterial promoters http://www.softberry.com/ berry.phtml. The gene organization of the $f p g$ flanking regions in different species was compared using the String program http://string.embl.de/.

\section{Purification of the recombinant Mc MI080 Fpg protein}

E. coli strain ER2566 over-expressing Mc Fpg encoded by the plasmid pET22b-fpgM1080 was grown in LB medium containing ampicillin to a final concentration of $100 \mu \mathrm{g} /$ $\mathrm{ml}$ at $37^{\circ} \mathrm{C}$ with shaking until $\mathrm{OD}_{600}$ was 0.6 . The cells were induced with $1 \mathrm{mM}$ isopropyl-D-thiogalactopyranoside and grown for 4 hours. Cells were harvested and washed in phosphate-buffered saline and stored at $-70^{\circ} \mathrm{C}$. The cells were resuspended in sonication buffer containing $50 \mathrm{mM} \mathrm{Na}_{2} \mathrm{HPO}_{4} / \mathrm{NaH}_{2} \mathrm{PO}_{4}, 300 \mathrm{mM} \mathrm{NaCl}, \mathrm{pH} 8.0$ and protease inhibitor complete without EDTA (Roche Applied Science, Germany) before lysis by sonication. The cleared lysate was loaded onto a Ni-NTA agarose column 
(Qiagen, Germany) and the column washed with wash buffer containing $20 \mathrm{mM}$ imidazole. Bound protein was eluted with a step gradient of 2 column volumes of the elution buffer containing 40, 60, 80, 100, 140, 180, 220 and $250 \mathrm{mM}$ imidazole. Fractions containing purified protein were pooled and dialysed against $25 \mathrm{mM}$ Tris$\mathrm{HCl}, \mathrm{pH} 7.5,300 \mathrm{mM} \mathrm{NaCl}$ and $10 \%$ glycerol.

\section{Assay for base excision of 8oxoG opposite $C, A, G$ or $T$} Duplex DNA substrates containing a single 8oxoG opposite of C, A, G or T were generated by ${ }^{32} \mathrm{P} 5^{\prime}$ end-labelling of oligonucleotides, using T4 polynucleotide kinase (New England Biolabs, MA) followed by annealing to a complementary oligonucleotide [20]. The oligonucleotide sequences of the DNA substrates are listed in Table 2. DNA glycosylase reactions were performed by mixing purified protein with $10-50 \mathrm{fmol}$ DNA substrate in a total volume of $10 \mu \mathrm{l}$. The enzyme activities were assayed in the reaction buffer previously described [20] and incubated at $37^{\circ} \mathrm{C}$ for 30 min. E. coli Fpg (New England Biolabs, MA) was included as a positive control. Products of the reactions were separated by $20 \%$ denaturing PAGE and visualized by phosphorimaging. The assay was performed in triplicate.

\section{Assay for formamidopyrimidine (faPy) DNA glycosylase activity}

$N$-[H3]-N-methyl- $N$ '-nitrosourea (MNU; $1.5 \mathrm{Ci} \mathrm{mmol}^{-1}$ ) was used to prepare poly(dG-dC) DNA $(12,000 \mathrm{dpm}$ mg1) [21]. DNA glycosylase activity was assayed by mixing purified protein with substrate in a reaction buffer containing $70 \mathrm{mM} 3$-( $\mathrm{N}$-morpholino) propane sulfonic acid, pH 7.5, 1 mM EDTA, $1 \mathrm{mM}$ dithiothreitol (DTT) and 5\% glycerol for $30 \mathrm{~min}$ at $37^{\circ} \mathrm{C}$. Removal of bases was measured in a total reaction volume of $50 \mu \mathrm{l}$ containing $14 \mu \mathrm{g}$ of DNA substrate and 500 ng of purified meningococcal protein or $160 \mathrm{U}$ of E. coli Fpg (New England Biolabs, $\mathrm{MA})$. The assay was repeated 5 times.

\section{Screening for phase variation by use of a universal rate of switching (UROS) cassette}

To promote efficient natural transformation, a 12-mer DNA uptake sequence was inserted into plasmid pARR2107 containing a Universal Rate of Switching (UROS) cassette (kind gift from H. L. Alexander, Emory University School of Medicine, Atlanta, GA) [22], creating plasmid pUD. Mc strain Z1099 (kind gift from D. A. Caugant, Norwegian Institute of Public Health, Oslo, Norway) was transformed with pUD and named NmZ1099_UROS. The mutS and fpg genes of NmZ1099_UROS were inactivated by insertion of a kanamycin resistance cassette as described by Davidsen et al., 2007 [9] in two separate genetic transformations creating strains NmZ1099_UROSAmutS and NmZ1099_UROS $\Delta f p g$. The mononucleotide tract of $8 \mathrm{G}$ residues preceding the spectinomycin resistance gene of the UROS cassette was confirmed as an intact 8-mer by PCR and sequencing (by using the primers listed in Table 2) in all three strains before switching frequency/phase variation was assessed. Briefly, Mc strains were grown overnight at $37^{\circ} \mathrm{C}, 5 \% \mathrm{CO}_{2}$, before 10 colonies were resuspended in GC broth. Serial dilutions were plated on GC agar with and without spectinomycin (to a final concentration of $50 \mathrm{mg} / \mathrm{l}$ ) and incubated overnight. The spectinomycin OFF to ON switching rate was determined by dividing the number of colonies on GC plates containing spectinomycin by the number of colonies on plain GC plates. Phase variation experiments were repeated at least 5 times for each strain. Significance in differences in phase variation frequency was calculated by the Kruskal-Wallis test.

\section{Results and discussion}

Fpg is nearly ubiquitous among bacterial species and is highly conserved both within annotated neisserial genome sequences and clinical Mc isolates [10], as well as between evolutionarily distant prokaryotes. We examined the activity and specificity of recombinant Mc Fpg purified to homogeneity towards representative substrates resulting from oxidative DNA damage, 8oxoG and faPy, and detected prototype Fpg glycosylase activity. Previously, we have shown a synergistic effect between the two GO components MutY and Fpg in Mc [9]. Together, these findings emphasize a distinct role for Fpg in the defense against the deleterious effects of reactive oxygen species.

The putative Mc fpg open reading frame (ORF) consists of 828 bp and contains a DNA uptake sequence (DUS) (5'GCCGTCTGAA-3') (Figure 1A). The Mc genome harbours approximately 2000 copies of this highly conserved $10 \mathrm{bp}$ sequence, which is required for efficient transformation [23]. A 12-mer DUS with two additional bp upstream of the core $10 \mathrm{bp}$ repeat element improves the transformation efficiency [24]. The Mc fpg gene contains one 11-mer. A single complete DUS or AT-DUS (10-, 11- or 12-mer) may promote the reacquisition of a gene by transformation if it is damaged or deleted and DUS occurs at higher densities in genome maintenance genes than in other house-keeping genes [25].

The organization of the $f p g$ flanking region is unique for Neisseria species http://string.embl.de/ (data not shown). Upstream of the $f p g$ gene are the hypothetical ORFs NMB1297 and NMB1296 (Figure 1A). NMB1297 is annotated as an ortholog to mltD http:// www.ncbi.nlm.nih.gov/COG/, which encodes a membrane-bound lytic murein transglycosylase of unknown function. NMB1296 shows 30-40\% amino acid identity with DNA methyltransferases in a number of bacterial species http://www.ncbi.nlm.nih.gov/blast/Blast.cgi. 


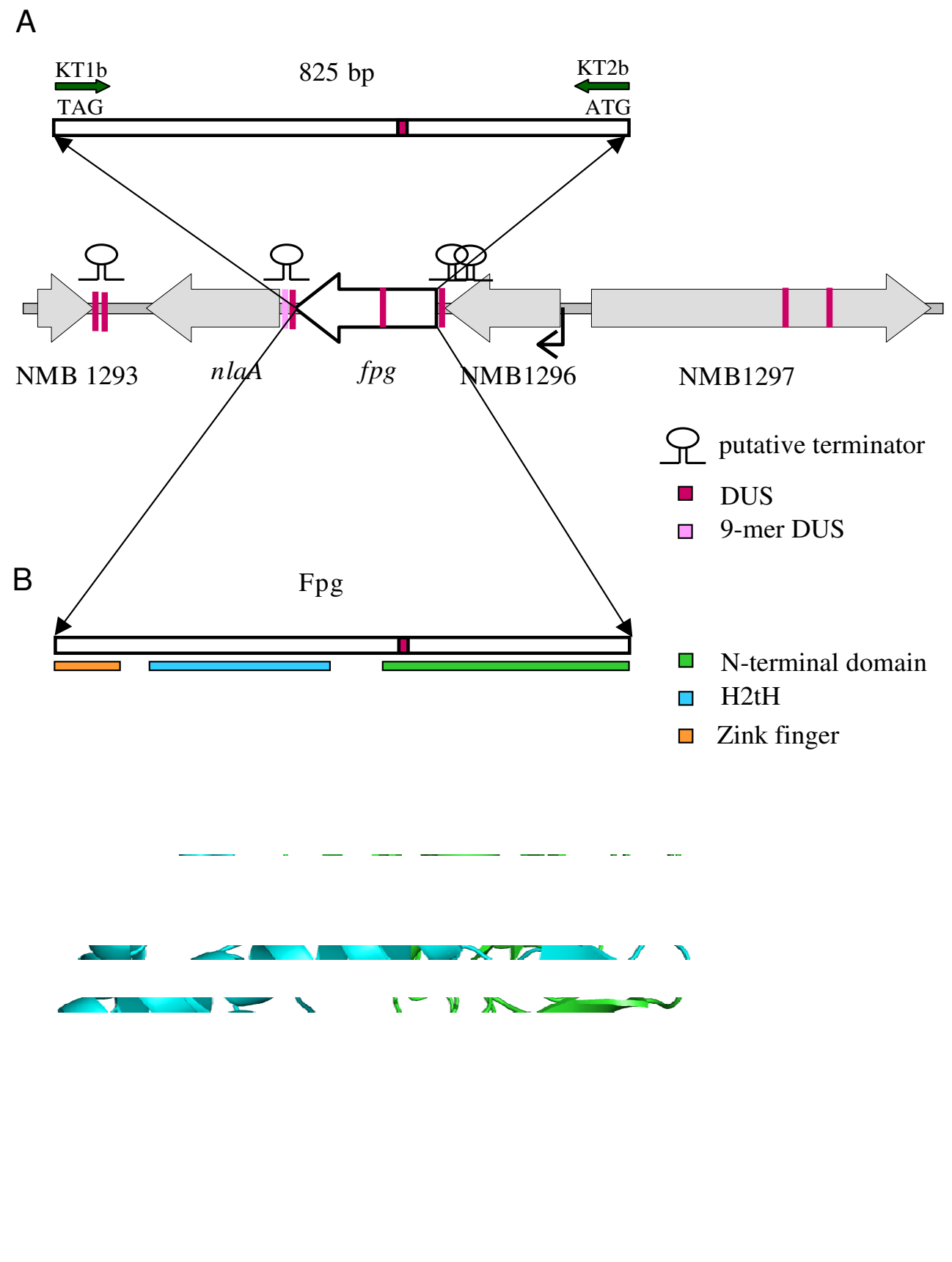

\section{Figure I}

N. meningitidis (Mc) Fpg. (A) Physical map of the Mc fpg open reading frame and flanking regions. The $f p g$ gene contains a DNA uptake sequence (DUS). Primers KTIb and KT2b employed in cloning of the Mc fpg gene are depicted. The gene organization of the Mc fpg flanking regions is identical in all available neisserial genomes. NMBI296 encodes a hypothetical protein with sequence homology to DNA methyltransferases. A promoter is predicted upstream of NMBI296 (black arrow). The fpg and the lysophophatidic acid acyltransferase nlaA genes are putatively co-transcribed [27], although an inverted repeat (containing DUS) associated with transcription termination or attenutation is found downstream of the fpg gene. NMBI297 is COGannotated mltD (membrane-bound lytic murein transglycosylase). NMBI 293 is a hypothetical protein. The distribution of DUS and degenerate DUS is indicated. (B) Structural modeling of Mc Fpg based on E. coli Fpg (PDB Ik82) showing the DNA binding motifs helix-two-turn-helix $(\mathrm{H} 2 \mathrm{tH})$ (blue) and zinc finger (orange), as well as the $\mathrm{N}$-terminal domain (green) containing the glycosylase catalytic amino acid residues. Amino acids encoded by DUS are highlighted in purple. 
Downstream, fpg is flanked by the nlaA gene, encoding a lysophosphatidic acid acyltransferase involved in biosynthesis of the glycerophosholipid membrane [26], about 300 bp of non-coding sequence containing two DUS within a predicted terminator, and the opposite oriented hypothetical ORF NMB1293. The NMB1296, fpg and nlaA genes are all oriented in the same direction and a putative promoter is found upstream of NMB1296 while none are identified between these genes. At the end of NMB1296 a terminator is predicted by TransTermHP. Between $f p g$ and $n l a A$, a terminator is predicted by GeSTer. This intrinsic terminator contains a DUS and an imperfect DUS as inverted repeat, a structure found in many putative Mc transcription terminators or attenuators [24]. The VIMSS Operon Prediction suggests co-transcription of $f p g$ and NMB1296. However, Swartley and Stephens have evidence by reverse transcriptase PCR that nlaA and $f p g$ are co-transcribed in Mc strain NMB [27]. In microarray analysis of an MC58 fpg mutant compared to wildtype, nlaA was the only gene significantly down-regulated at least 1.5 fold, supporting the evidence for co-transcription of these two genes (unpublished data).

The Mc fpg open reading frame encodes 276 amino acids containing a predicted $\mathrm{N}$-terminal glycosylase catalytic domain, a helix-two-turn-helix and a C-terminal zinc finger (Figure 1B, additional file 1, Figures S1 and S2). These regions contain long sequences with a positive electrostatic charge, enforcing binding to negatively charged DNA (See additional file 1, Figure S3). Alignment of the deduced Fpg sequence from the genomes of five Mc strains reveals non-synonymous or synonymous substitutions in 5 out of 276 amino acid positions (see additional file 1, Figure S1). The positions showing variation correspond exactly to those found in the fpg gene from $11 \mathrm{Mc}$ clinical isolates previously sequenced [10]. An additional 6 amino acids show non-synonymous or synonymous variation when the $N$. gonorrhoeae and $N$. lactamica sequences are included in the comparison. All known functional residues exhibit complete sequence conservation (see additional file 1, Table S1 and Figure S1). Comparison of the neisserial Fpg sequences to those in species where the Fpg crystal structure is solved [28-31] also shows a high degree of conservation, especially in the functional domains and catalytic amino acid residues (see additional file 1, Figure S2). This conservation was confirmed by in silico fusion of the crystal structure of Lactococcus lactis Fpg with Mc Fpg using the PDB (Figure 1B). Interestingly, the 11-mer DUS sequence encodes amino acids that are not identified as functional residues and is localized in an $f p g$ region showing relatively low sequence homology across species boundaries (see additional file 1 , Figures S1 and S2).

Fpg has been extensively studied in E. coli and is characterized in several other prokaryotes as well [32-34], display- ing identical substrate specificities. In order to analyze the substrate specificity of $\mathrm{Mc}$ Fpg, the gene was overexpressed in E. coli and recombinant Mc Fpg protein purified to homogeneity (see additional file 1, Figure S4). Mc Fpg has an apparent size in SDS-PAGE of approximately $30 \mathrm{kDa}$, corresponding to the molecular weight predicted from the genome deduced amino acid sequence and similar to Fpg of E. coli and L. lactis [32,33]. The preferred substrates for recognized Fpg proteins are 8oxoG and faPy residues. The ability of recombinant Mc Fpg to remove these lesions was investigated, using E. coli Fpg as a positive control. Activity towards C:faPy residues in a ${ }^{3} \mathrm{H}$ labeled poly(dG-dC) substrate was identified (Table 3). When assessing the 8oxoG excision, the Mc Fpg displayed both DNA glycosylase and AP lyase activity (Figure 2). Equivalent levels of base excision of 8oxoG opposite C, T and $\mathrm{G}$ and much lower activity toward $80 x 0 \mathrm{G}$ when mispaired with A was demonstrated (Figure 2). No activity was dectected in the absence of 8 oxoG residues (see additional file 1, Figure S5). This discrimination of the base opposite the lesion is in keeping with findings on E. coli Fpg [35], although the remaining activity against 8oxoG:A seen in Mc Fpg was not found in the original characterization of substrate specificity in E. coli. 8oxoG:C is probably the most important physiological substrate for Mc Fpg, despite the similar levels of nicking observed in 8oxoG:T and 8oxoG:G, as the former is by far the most common substrate in vivo in E. coli [4]. The removal of 8oxoG from the genome prevents $\mathrm{G}: \mathrm{C} \rightarrow \mathrm{T}: \mathrm{A}$ transversions in E. coli, but the mutation rates in single fpg mutants are too low in $\mathrm{Mc}$ to detect these lesions [9], despite this being the most likely event when 8oxoG is preferentially mis-incorporated with adenine and left unrepaired. Recent studies in M. smegmatis have identified an alternative pattern of preferential incorporation of guanine opposite $80 x o G$, creating $\mathrm{G}: \mathrm{C} \rightarrow \mathrm{C}: \mathrm{G}$ transversions or $\mathrm{A}: \mathrm{T} \rightarrow \mathrm{C}: \mathrm{G}$ transitions in the absence of Fpg [36]. 8oxoG:G and G:C $\rightarrow$ C:G transversions can also be found in E. coli and $S$. pombe, however, they are rare compared to 8oxoG:A events. In conclusion, these results demonstrate that the protein encoded by the Mc fpg gene excises base lesions that are typical substrates of other Fpg orthologues and are consistent with this protein being an Fpg DNA glycosylase.

Mc is a bacterium that seemingly spontaneously produces a plethora of variants upon which selection can act, instead of sensing the environment and changing accordingly [37]. One of the major processes governing genetic changes in Neisseria sp. is phase variation. Phase variation is mediated by unstable polynucleotide tracts allowing the gene expression to be switched on or off [37]. Recently, several genome maintenance genes have been shown to modulate phase variation frequencies, including the mismatch repair components mutS and $m u t L$, the nucleotide excision repair gene $u v r D$ and the translesion DNA polymerase $\operatorname{din} B$ [38-41]. Since Mc Fpg is able to remove 
A

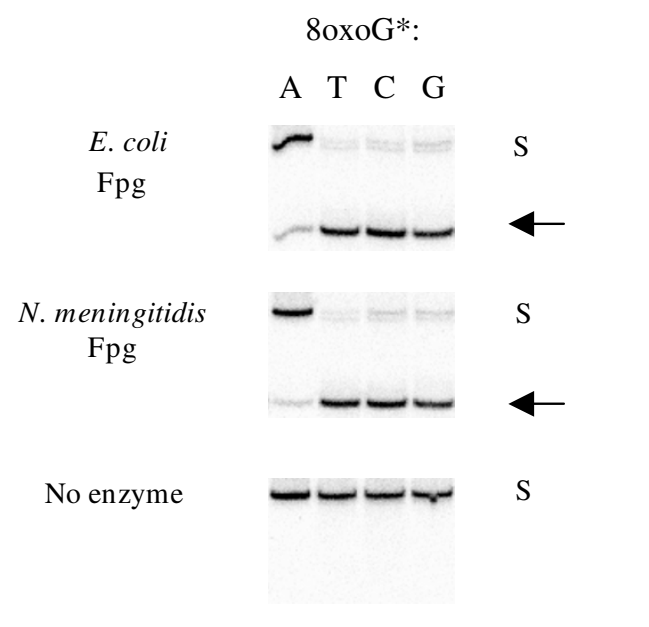

B

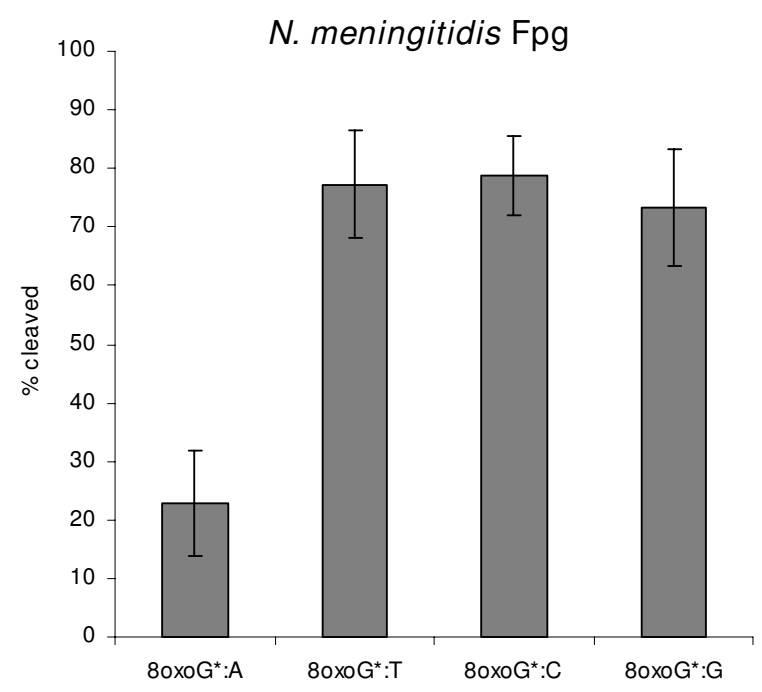

Figure 2

DNA glycosylase activity of $\boldsymbol{N}$. meningtidis (Mc) recombinant Fpg protein. (A) I ng of purified Mc Fpg or 0.032 Units of $E$. coli Fpg was incubated with $10-50 \mathrm{fmol}$ of a 24 bp duplex oligodeoxyribonucleotide containing a single 8oxoG residue opposite $A, T C$ or $G$. Base excision and strand cleavage were analysed by $20 \%$ PAGE and phosphorimaging. The arrow indicates the cleaved DNA substrate. * denotes ${ }^{32}$ P-labelled strand. S; substrate. (B) Quantification of strand cleavage activity by Mc Fpg. The results represent the average of three independent experiments and error bars indicate the standard deviation of the mean.

oxidized guanines, although in an error-free manner, we wanted to investigate a potential contribution of Mc fpg on phase variation of polyG tracts. Mc strains
Table 3: DNA glycosylase activity of $\boldsymbol{N}$. meningitidis (Mc) recombinant Fpg protein.

\begin{tabular}{lll}
\hline Substrate & \multicolumn{2}{l}{ Released bases (fmol) } \\
\cline { 2 - 3 } & Average & $(\text { St. dev. })^{\mathrm{c}}$ \\
\hline N. meningitidis Fpga & 75 & $( \pm 30)$ \\
E. coli Fpgb & 64 & $( \pm 44)$ \\
No enzyme & 12 & $( \pm 4)$ \\
\hline
\end{tabular}

a $500 \mathrm{ng}$ of protein was employed in each reaction

b I 60 Units of protein was employed in each reaction

c standard deviation of the mean

Removal of formamidopyrimidine (faPy) from [3H]-methyl-faPypoly $(\mathrm{dG} \cdot \mathrm{dC})$ DNA by recombinant Mc and E. coli Fpg. The results are given as the average of five independent measurements.

NmZ1099_UROS (Control), NmZ1099_UROS $\triangle f p g$ $(\Delta f p g)$ and NmZ1099_UROS $\Delta m u t S$ ( $\Delta m u t S)$ were constructed and examined by S12 ribosomal gene switching in a spectinomycin-selection assay (Figure 3 ). Phase variation was, as previously reported [38-41], significantly increased in the $\Delta m u t S$ (30-fold) background compared to the wild-type level $\left({ }^{* * *} \mathrm{p}<0.001\right)$. However, the Mc $f p g$ mutant exhibited only moderate increase (2-fold) compared to the wild-type level $\left({ }^{* * *} \mathrm{p}<0.001\right)$, and thus MutS exerts a more profound effect on the stability of Mc polyG tracts than Fpg. Likewise, the Mc fpg mutant was recently shown to generate only a weak mutator phenotype when assessed for its spontaneous mutation frequency in a rifampicin assay [9]. In conclusion, Fpg is not a major player in modulating Mc mutation frequencies.

Although Mc Fpg displays traits characteristic of the Fpg family of proteins, survival rates of a Mc fpg mutant were not affected by exposure to reactive oxygen species [9]. This is in contrast to findings in M. smegmatis, where $\mathrm{H}_{2} \mathrm{O}_{2}$ exposure proved to be lethal to fpg null mutants [36], and in the photosynthetic cyanobacteria $S$. elongates where an $f p g$-deficient strain exhibited progressively reduced survival with increasing levels of oxidatively damaging irradiation [42]. Considering the potential importance of oxidative DNA damage in the Mc habitat combined with the vulnerability of a relatively $\mathrm{G}+\mathrm{C}$ rich genome obtaining such lesions, the explanation for the species discrepancy should be investigated further. The Fpg family of DNA glycosylases also contains endonuclease VIII (Nei) and eukaryotic Nei orthologues. The Nei proteins excise oxidized pyrimidines and may also serve as a backup for removal of 8oxoG in E. coli [43], however, no Mc Nei ortholog has been identified $[11,15]$. On the other hand, the abundant Mc anti-oxidant system provides particularly high protection towards the generation of such DNA lesions [44]. In general, the elucidation of the Mc DNA repair profile is important for understanding the lifestyle 


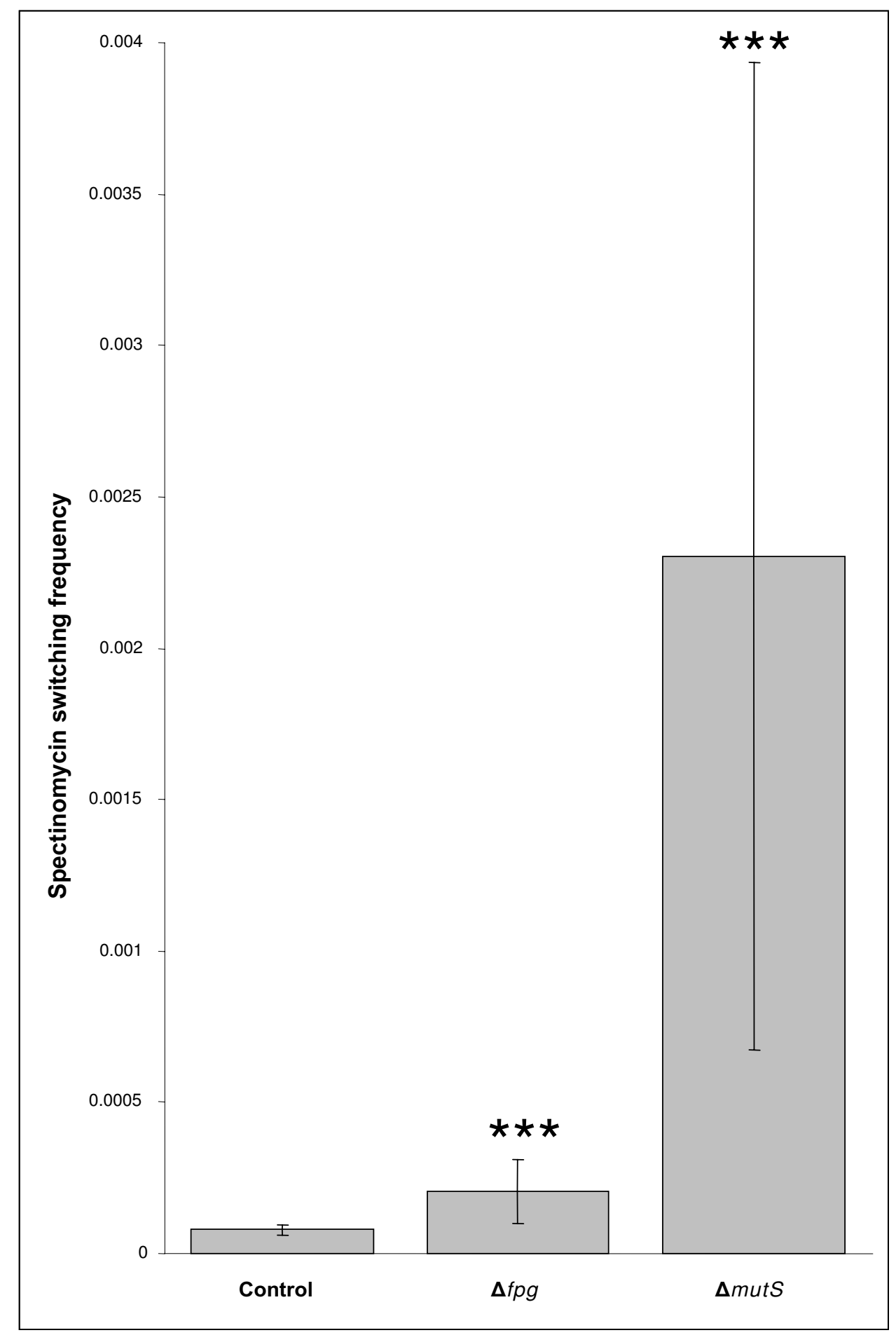

Figure 3

Assessment of meningococcal (Mc) phase variation. Phase variation frequency for Mc strains NmZI099_UROS (Con-

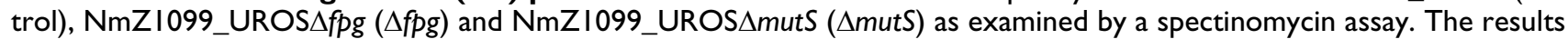
are given as the median of at least 5 independent measurements. Error bars represent \pm I quartile. Phase variation is moderately and significantly increased, respectively, in the Mc $\Delta f p g$ (2-fold) and $\Delta$ mutS (30-fold) background compared to the wildtype level $(* * * 0<0.001)$. 
of this important pathogen, commensal and model organism.

\section{Conclusion}

Mc fpg contains DUS both within its coding sequence and in close proximity to the open reading frame, potentially promoting reacquisition of this gene by transformation if it is damaged or lost. The fpg gene may belong to an operon together with a putative DNA methyltransferase and a lysophosphatidic acid acyltransferase, although the reasons for this gene organisation remain obscure. Both the nucleotide and amino acid sequences of neisserial Fpg homologues are highly conserved. In addition, Mc Fpg amino acid sequence shows great conservation across species boundaries in functional domains, and Mc Fpg contains a predicted N-terminal glycosylase catalytic domain, a helix-two-turn-helix and a C-terminal zinc finger. Accordingly, Mc Fpg exhibits DNA glycosylase and AP lyase activities and remove both 8oxoG and faPy lesions. When examining the stability of polyG tracts, MutS was found to modulate mutation frequencies due to phase variation to a much higher extent than Fpg. In conclusion, Mc Fpg predicted structure and activity pattern were found to be similar to those of prototype Fpg orthologues in other species. Together, these findings emphasize a distinct role for Mc Fpg in the defense against the deleterious effects of reactive oxygen species.

\section{Authors' contributions}

KLT carried out the molecular genetic studies and analysis of purified protein, performed sequence alignments and drafted the manuscript. OHA constructed pUD, designed the phase variation studies and performed the GeSTer analysis. KA contributed to pUD construction and performed the phase variation studies. HH purified recombinant proteins. SAF participated in the bioinformatic analyses. TD supervised the molecular studies and analysis of purified protein, and assisted in manuscript writing. TT conceived the study, participated in its design and coordination and drafted the manuscript. All authors read and approved the final manuscript.

\section{Additional material}

\section{Additional file 1}

Supplementary Material. contains Table S1 Deduced amino acid sequence of Fpg homologues in Neisseria, Figure S1 Deduced amino acid sequence of Fpg homologues in Neisseria, Figure S2 Deduced amino acid sequence of Fpg orthologues, Figure S3 Electrostatic charge of meningococcal Fpg, Figure S4 Purified meningococcal Fpg, Figure S5 Meningococcal Fpg activity towards undamaged DNA substrate.

Click here for file

[http://www.biomedcentral.com/content/supplementary/1471-

2180-9-7-S1.doc]

\section{Acknowledgements}

The Medical Research Curriculum at the University of Oslo is greatly acknowledged for its support to KLT. This work was also supported by grants from the Research Council of Norway.

\section{References}

I. Yazdankhah SP, Caugant DA: Neisseria meningitidis: an overview of the carriage state. J Med Microbiol 2004, 53:82I-832.

2. Stephens DS, Greenwood B, Brandtzaeg P: Epidemic meningitis, meningococcaemia, and Neisseria meningitidis. Lancet 2007, 369:2196-2210.

3. O'Rourke EJ, Chevalier C, Pinto AV, Thiberge JM, lelpi L, Labigne A, Radicella JP: Pathogen DNA as target for host-generated oxidative stress: role for repair of bacterial DNA damage in Helicobacter pylori colonization. Proc Natl Acad Sci USA 2003, 100:2789-2794.

4. Cheng KC, Cahill DS, Kasai H, Nishimura S, Loeb LA: 8-Hydroxyguanine, an abundant form of oxidative DNA damage, causes G-T and A-C substitutions. I Biol Chem 1992, 267:166-I72.

5. Boiteux S, Laval J: Imidazole open ring 7-methylguanine: an inhibitor of DNA synthesis. Biochem Biophys Res Commun 1983, I 1 0:552-558.

6. Bjelland S, Seeberg E: Mutagenicity, toxicity and repair of DNA base damage induced by oxidation. Mutat Res 2003, $53 \mathrm{I}: 37-80$.

7. Bhagwat M, Gerlt JA: 3 '- and 5 '-strand cleavage reactions catalyzed by the Fpg protein from Escherichia coli occur via successive beta- and delta-elimination mechanisms, respectively. Biochemistry (Mosc) 1996, 35:659-665.

8. Michaels ML, Miller JH: The GO system protects organisms from the mutagenic effect of the spontaneous lesion 8hydroxyguanine (7,8-dihydro-8-oxoguanine). J Bacteriol 1992, 174:632|-6325.

9. Davidsen T, Tuven HK, Bjoras M, Rodland EA, Tonjum T: Genetic interactions of DNA repair pathways in the pathogen Neisseria meningitidis. J Bacteriol 2007, 189:5728-5737.

10. Davidsen T, Amundsen EK, Rodland EA, Tonjum T: DNA repair profiles of disease-associated isolates of Neisseria meningitidis. FEMS Immunol Med Microbiol 2007, 49:243-25I.

II. Tettelin H, Saunders NJ, Heidelberg J, Jeffries AC, Nelson KE, Eisen JA, Ketchum KA, Hood DW, Peden JF, Dodson RJ, et al.: Complete genome sequence of Neisseria meningitidis serogroup B strain MC58. Science 2000, 287:1809-1815.

12. Sambrook J, Russell DW: Molecular cloning: a laboratory manual. Cold Springs Laboratory Press. Cold Spring Harbor, New York; 2001.

13. Hulo N, Sigrist CJ, Le SV, Langendijk-Genevaux PS, Bordoli L, Gattiker A, De CE, Bucher P, Bairoch A: Recent improvements to the PROSITE database. Nucleic Acids Res 2004, 32:DI 34-DI 37.

14. Bateman A, Coin L, Durbin R, Finn RD, Hollich V, Griffiths-Jones S, Khanna A, Marshall M, Moxon S, Sonnhammer EL, et al:: The Pfam protein families database. Nucleic Acids Res 2004, 32:D I38-D I 4 I.

15. Parkhill J, Achtman M, James KD, Bentley SD, Churcher C, Klee SR, Morelli G, Basham D, Brown D, Chillingworth T, et al.: Complete DNA sequence of a serogroup A strain of Neisseria meningitidis Z249 I. Nature 2000, 404:502-506.

16. Bentley SD, Vernikos GS, Snyder LA, Churcher C, Arrowsmith C, Chillingworth T, Cronin A, Davis PH, Holroyd NE, Jagels K, et al.: Meningococcal genetic variation mechanisms viewed through comparative analysis of serogroup C strain FAMI 8. PLoS Genet 2007, 3:e23.

17. Peng J, Yang L, Yang F, Yang J, Yan Y, Nie H, Zhang X, Xiong Z, Jiang $Y$, Cheng F, et al:: Characterization of ST-482I complex, a unique Neisseria meningitidis clone. Genomics 2008, 91 1:78-87.

18. Cuff JA, Clamp ME, Siddiqui AS, Finlay M, Barton GJ: JPred: a consensus secondary structure prediction server. Bioinformatics 1998, 14:892-893.

19. Price MN, Huang KH, Alm EJ, Arkin AP: A novel method for accurate operon predictions in all sequenced prokaryotes. Nucleic Acids Res 2005, 33:880-892.

20. Eide L, Bjoras M, Pirovano M, Alseth I, Berdal KG, Seeberg E: Base excision of oxidative purine and pyrimidine DNA damage in Saccharomyces cerevisiae by a DNA glycosylase with sequence similarity to endonuclease III from Escherichia coli. Proc Natl Acad Sci USA 1996, 93:10735-10740. 
21. Boiteux S, Belleney J, Roques BP, Laval J: Two rotameric forms of open ring 7-methylguanine are present in alkylated polynucleotides. Nucleic Acids Res 1984, I 2:5429-5439.

22. Alexander HL, Richardson AR, Stojiljkovic I: Natural transformation and phase variation modulation in Neisseria meningitidis. Mol Microbiol 2004, 52:77I-783.

23. Goodman SD, Scocca J]: Identification and arrangement of the DNA sequence recognized in specific transformation of Neisseria gonorrhoeae. Proc Natl Acad Sci USA 1988, 85:6982-6986.

24. Ambur $\mathrm{OH}$, Frye SA, Tonjum T: New functional identity for the DNA uptake sequence in transformation and its presence in transcriptional terminators. J Bacteriol 2007, I 89:2077-2085.

25. Davidsen T, Rodland EA, Lagesen K, Seeberg E, Rognes T, Tonjum T: Biased distribution of DNA uptake sequences towards genome maintenance genes. Nucleic Acids Res 2004, 32:1050-1058.

26. Swartley JS, Balthazar JT, Coleman J, Shafer WM, Stephens DS: Membrane glycerophospholipid biosynthesis in Neisseria meningitidis and Neisseria gonorrhoeae: identification, characterization, and mutagenesis of a lysophosphatidic acid acyltransferase. Mol Microbiol 1995, 18:40 I-4I2.

27. Swartley JS, Stephens DS: Co-transcription of a homologue of the formamidopyrimidine-DNA glycosylase $(\mathrm{fpg})$ and lysophosphatidic acid acyltransferase (nlaA) in Neisseria meningitidis. FEMS Microbiol Lett 1995, 134: I7I-176.

28. Sugahara M, Mikawa T, Kumasaka T, Yamamoto M, Kato R, Fukuyama $K$, Inoue Y, Kuramitsu S: Crystal structure of a repair enzyme of oxidatively damaged DNA, MutM (Fpg), from an extreme thermophile, Thermus thermophilus HB8. EMBO J 2000, 19:3857-3869.

29. Serre L, Pereira de JK, Boiteux S, Zelwer C, Castaing B: Crystal structure of the Lactococcus lactis formamidopyrimidineDNA glycosylase bound to an abasic site analogue-containing DNA. EMBO / 2002, $21: 2854-2865$.

30. Gilboa R, Zharkov DO, Golan G, Fernandes AS, Gerchman SE, Matz E, Kycia JH, Grollman AP, Shoham G: Structure of formamidopyrimidine-DNA glycosylase covalently complexed to DNA. J Biol Chem 2002, 277: 1981 I-19816.

31. Fromme JC, Verdine GL: Structural insights into lesion recognition and repair by the bacterial 8-oxoguanine DNA glycosylase MutM. Nat Struct Biol 2002, 9:544-552.

32. Boiteux S, O'Connor TR, Lederer F, Gouyette A, Laval J: Homogeneous Escherichia coli FPG protein. A DNA glycosylase which excises imidazole ring-opened purines and nicks DNA at apurinic/apyrimidinic sites. / Biol Chem 1990, 265:3916-3922.

33. Duwat P, de OR, Ehrlich SD, Boiteux S: Repair of oxidative DNA damage in gram-positive bacteria: the Lactococcus lactis Fpg protein. Microbiology 1995, I4I(Pt 2):4I I-4I7.

34. Senturker S, Bauche C, Laval J, Dizdaroglu M: Substrate specificity of Deinococcus radiodurans Fpg protein. Biochemistry (Mosc) 1999, 38:9435-9439.

35. Tchou J, Kasai H, Shibutani S, Chung MH, Laval J, Grollman AP, Nishimura S: 8-oxoguanine (8-hydroxyguanine) DNA glycosylase and its substrate specificity. Proc Natl Acad Sci USA 1991, 88:4690-4694.

36. Jain R, Kumar P, Varshney U: A distinct role of formamidopyrimidine DNA glycosylase (MutM) in down-regulation of accumulation of $\mathbf{G}, \mathbf{C}$ mutations and protection against oxidative stress in mycobacteria. DNA Repair (Amst) 2007, 6: 1774-1785.

37. Moxon ER, Rainey PB, Nowak MA, Lenski RE: Adaptive evolution of highly mutable loci in pathogenic bacteria. Curr Biol 1994, 4:24-33.

38. Richardson AR, Stojiljkovic I: Mismatch repair and the regulation of phase variation in Neisseria meningitidis. Mol Microbiol 200I, 40:645-655

39. Richardson AR, Yu Z, Popovic T, Stojiljkovic I: Mutator clones of Neisseria meningitidis in epidemic serogroup A disease. Proc Natl Acad Sci USA 2002, 99:6103-6107.

40. Alexander HL, Rasmussen AW, Stojiljkovic I: Identification of Neisseria meningitidis genetic loci involved in the modulation of phase variation frequencies. Infect Immun 2004, 72:6743-6747.

41. Martin P, Sun L, Hood DW, Moxon ER: Involvement of genes of genome maintenance in the regulation of phase variation frequencies in Neisseria meningitidis. Microbiology 2004, I 50:300I-30I2.
42. Muhlenhoff U: The FAPY-DNA glycosylase $(F p g)$ is required for survival of the cyanobacterium Synechococcus elongatus under high light irradiance. FEMS Microbiol Lett 2000, 187:127-132.

43. Blaisdell JO, Hatahet Z, Wallace SS: A novel role for Escherichia coli endonuclease VIII in prevention of spontaneous G-->T transversions. J Bacteriol 1999, I 81:6396-6402.

44. Seib KL, Tseng HJ, McEwan AG, Apicella MA, Jennings MP: Defenses against oxidative stress in Neisseria gonorrhoeae and Neisseria meningitidis: distinctive systems for different lifestyles. J Infect Dis 2004, 190:136-147.

45. Frasch CE, Gotschlich EC: An outer membrane protein of Neisseria meningitidis group $B$ responsible for serotype specificity. J Exp Med 1974, 140:87-104.
Publish with Bio Med Central and every scientist can read your work free of charge

"BioMed Central will be the most significant development for disseminating the results of biomedical research in our lifetime. "

Sir Paul Nurse, Cancer Research UK

Your research papers will be:

- available free of charge to the entire biomedical community

- peer reviewed and published immediately upon acceptance

- cited in PubMed and archived on PubMed Central

- yours - you keep the copyright 